\title{
PKG-mediated MAPK signaling is necessary for long-term operant memory in Aplysia
}

\author{
Maximilian Michel, ${ }^{1,3}$ Charity L. Green, ${ }^{1,2,3}$ Arnold Eskin, $^{2}$ and Lisa C. Lyons ${ }^{1,2,4}$ \\ ${ }^{1}$ Department of Biological Science, Program in Neuroscience, Florida State University, Tallahassee, Florida 32306, USA; ${ }^{2}$ Department \\ of Biology and Biochemistry, University of Houston, Houston, Texas 77204, USA
}

\begin{abstract}
Signaling pathways necessary for memory formation, such as the mitogen-activated protein kinase (MAPK) pathway, appear highly conserved across species and paradigms. Learning that food is inedible (LFI) represents a robust form of associative, operant learning that induces short- (STM) and long-term memory (LTM) in Aplysia. We investigated the role of MAPK signaling in LFI memory in vivo. Inhibition of MAPK activation in animals prior to training blocked STM and LTM. Discontinuing MAPK signaling immediately after training inhibited LTM with no impact on STM. Therefore, MAPK signaling appears necessary early in memory formation for STM and LTM, with prolonged MAPK activity required for LTM. We found that LFI training significantly increased phospho-MAPK levels in the buccal ganglia. Increased MAPK activation was apparent immediately after training with greater than basal levels persisting for $2 \mathrm{~h}$. We examined the mechanisms underlying training-induced MAPK activation and found that PKG activity was necessary for the prolonged phase of MAPK activation, but not for the early MAPK phase required for STM. Furthermore, we found that neither the immediate nor the prolonged phase of MAPK activation was dependent upon nitric oxide (NO) signaling, although expression of memory was dependent on NO as previously reported. These studies emphasize the role of MAPK and PKG in negatively reinforced operant memory and demonstrate a role for PKG-dependent MAPK signaling in invertebrate associative memory.
\end{abstract}

[Supplemental material is available for this article.]

Invertebrate model systems with relatively simple nervous systems have proven invaluable in furthering our understanding of adaptive behavior and the mechanisms underlying memory, as core components appear highly conserved across phylogenies (reviewed in Bailey et al. 2008; Sweatt 2010). One conserved element, the mitogen-activated protein kinase cascade (MAPK) is necessary for intermediate and long-term sensitization in Aplysia (Martin et al. 1997; Purcell et al. 2003; Sharma et al. 2003), olfactory conditioning in Drosophila (Moressis et al. 2009; Pagani et al. 2009), and associative memory in mammals (reviewed in Sweatt 2004). Recently, the nitric oxide-cGMP-Protein kinase $\mathrm{G}$ (NO-cGMP-PKG) pathway was identified upstream of MAPK activation during fear conditioning in mammals (Ota et al. 2008, 2010b). In Aplysia, long-term hyperexcitability of nociceptive neurons following injury requires $\mathrm{NO}-\mathrm{cGMP}-\mathrm{PKG}$ signaling (Lewin and Walters 1999) for MAPK activation and nuclear translocation (Sung et al. 2004). Thus, the possibility exists that the PKG-MAPK pathway also may be conserved in invertebrate learning. However, no studies have addressed a PKGMAPK link during associative memory formation in invertebrates.

Feeding behavior in Aplysia and its underlying circuitry represent a highly plastic system (reviewed in Elliott and Susswein 2002; Cropper et al. 2004) amenable for comparative studies of complex learning through classical conditioning, operant reward learning, and negatively reinforced paradigms (reviewed in Baxter and Byrne 2006). Learning that food is inedible (LFI) is an operant paradigm during which an animal's failure to swallow a netted

\footnotetext{
${ }^{3}$ These authors contributed equally to this work.

${ }^{4}$ Corresponding author.

E-mail lyons@bio.fsu.edu; fax (850) 644-0989.

Article is online at http://www.learnmem.org/cgi/doi/10.1101//m.2063611.
}

seaweed provides negative reinforcement (Susswein and Schwarz 1983; Susswein et al. 1986). While considerable research exists on the behavioral parameters influencing LFI memory including circadian modulation of long-term memory (LTM)(Lyons et al. 2005), the effect of conspecifics, and the negative consequences of social isolation (Schwarz and Susswein 1992; Schwarz et al. 1998), relatively little is known regarding the molecular pathways underlying LFI memory. Recently, Protein Kinase A and Protein Kinase $\mathrm{C}$ were found necessary for long-term LFI memory formation (Michel et al. 2011), but no kinase pathway has been implicated in short-term LFI. In vivo, LFI also requires NO signaling for short-term memory (STM) and LTM (Katzoff et al. 2002). Although the precise role of NO remains unsettled, NO-cGMP signaling appears to convey failed swallowing information following reinforcement signals from the gut (Katzoff et al. 2002, 2010). Additional studies are needed on the mechanisms underlying LFI memory as feeding behavior plasticity represents a good system for the comparative analysis of adaptive behavior.

To further understand the molecular mechanisms underlying LFI memory, we investigated the role of MAPK and PKG signaling in STM and LTM in vivo. We found that MAPK signaling is necessary for the induction of STM and LTM, but not recall. We determined the temporal and spatial parameters of MAPK signaling and found that LFI training induced MAPK activation in the buccal ganglia immediately after training and for a prolonged period lasting $2 \mathrm{~h}$. Furthermore, LTM required prolonged MAPK signaling dependent, at least in part, upon PKG and seemingly independent of NO synthesis. Thus, PKG emerges as a critical step upstream of MAPK for LTM. These studies emphasize the role of MAPK and PKG in LFI memory and underscore important distinctions between nonassociative memory, operant reward learning, and negatively reinforced memory. 


\section{Materials and Methods}

\section{Animal maintenance}

Aplysia californica (Alacrity; Charles Hollahan) weighing 100$200 \mathrm{~g}$, were housed in individual boxes in 100 gallon tanks of artificial seawater (ASW; Instant Ocean, Aquarium Systems) at $15^{\circ} \mathrm{C}$ in 12 -h light:12-h dark cycles. Animals were fed romaine lettuce every second day until they were fed to satiation. All experiments were performed at $15^{\circ} \mathrm{C}$. To eliminate variance due to circadian modulation of long-term LFI (Lyons et al. 2005), all experiments were performed $3 \mathrm{~h}$ after the start of the light cycle at Zeitgeber Time (ZT) 3.

\section{Behavioral training and testing}

Animals were fed to satiation with laver seaweed and then food was withheld for 5-7 d before training. LFI training was done using protocols originally established by A. Susswein (Susswein et al. 1986; Botzer et al. 1998; Katzoff et al. 2002) and modified as previously reported (Lyons et al. 2005, 2006b). LFI training consisted of a one-trial training paradigm in which animals were presented with a small piece of laver seaweed wrapped in netting (tulle) approximately 3-4 $\mathrm{mm} \times 3-4 \mathrm{~mm}$ that could not be swallowed. The size of the netted seaweed varied slightly depending upon the size of the animals. Upon presentation of the netted seaweed, animals responded by head waving, biting, and attempts to swallow the netted seaweed. Training continued until the animal stopped responding to the netted seaweed for $3 \mathrm{~min}$. Two parameters were recorded: (1) the total time that the animals responded and (2) the cumulative time that the animal retained the netted seaweed in its mouth. Testing of the animals occurred using the same procedure either $30 \mathrm{~min}$ later for STM or $24 \mathrm{~h}$ later for LTM. Memory was represented as a decrease in total response time and the time the food was retained in the mouth. Naïve animals were handled similarly to trained animals.

\section{Drug treatments}

Two inhibitors of MAP kinase kinase (MEK) were used to inhibit MAPK signaling, U0126 (EMD Biosciences/Calbiochem; $130 \mu \mathrm{L} /$ $100 \mathrm{~g}$ body weight of $7.5 \mathrm{mM}$ stock in DMSO, approximates $15 \mu \mathrm{M}$ in the whole animal) and PD98059 (EMD Biosciences/ Calbiochem; $130 \mu \mathrm{L} / 100 \mathrm{~g}$ body weight of $15 \mathrm{mM}$ stock in DMSO, approximates $30 \mu \mathrm{M}$ in the whole animal). Control animals were injected with matched amounts of an inactive analog of U0126, U0124 $(130 \mu \mathrm{L} / 100 \mathrm{~g}$ body weight of $7.5 \mathrm{mM}$ stock, estimated systemic concentration $15 \mu \mathrm{M}$ in the animal) or DMSO. To inhibit PKG signaling, animals were injected with the specific inhibitor KT5823 (EMD Biosciences/Calbiochem; $1 \mathrm{~mL}$, $6.5 \mu \mathrm{M} / 100 \mathrm{~g}$ weight, estimated systemic concentration $100 \mathrm{nM}$ ) in ASW. L-NAME $(10 \mathrm{mg} / \mathrm{mL}$ per $100 \mathrm{~g}$ body weight, estimated systemic drug concentration $570 \mathrm{nM}$ ) injected $10 \mathrm{~min}$ prior to training was used to inhibit NO synthase.

\section{Western blotting}

Aplysia were anesthetized by injection of isotonic $\mathrm{MgCl}_{2}$ and ganglia dissected after LFI training. Ganglia were immediately homogenized in lysis buffer (50 mM Tris-HCl, pH 7.6, $150 \mathrm{mM} \mathrm{NaCl}, 2 \%$ SDS, $1 \mathrm{mM}$ EDTA, $1 \mathrm{mM}$ EGTA, $1 \mathrm{mM}$ sodium orthovanadate, $50 \mathrm{mM}$ sodium fluoride, and a protease inhibitor cocktail). Samples were boiled for $2 \mathrm{~min}$, centrifuged at $16,000 \mathrm{~g}$, and the supernatant was stored at $-80^{\circ} \mathrm{C}$. Proteins were resolved via $12 \%$ SDS-PAGE and transferred to PVDF membranes. Western blotting for P-MAPK and total MAPK was done as previously described (Sharma and Carew 2002; Sharma et al. 2003). Antibodies for P-MAPK (Cell Signaling) and total MAPK (Erk-1; Santa Cruz Biotechnology) were used. Quantification of P-MAPK was normalized to total MAPK.

\section{Statistics}

As denoted on the figure legends, the data was analyzed using a two-tailed Student's $t$-test or a one-way ANOVA as appropriate.
Bonferroni's post-hoc analysis was used for between group comparisons. $P$ values $<0.05$ were considered significant. Statistical analysis was performed using GraphPad Prism 4.00 (GraphPad Software).

\section{Results}

\section{MAPK signaling is necessary for long-term LFI memory, but not recall}

MAPK signaling comprises a necessary component of long-term memory for numerous learning paradigms, including nonassociative long-term facilitation and sensitization in Aplysia (Martin et al. 1997), long-term potentiation (English and Sweatt 1997), spatial learning in mammals (Selcher et al. 1999), and fear conditioning (Atkins et al. 1998; Schafe et al. 2000). To determine if MAPK signaling was necessary for long-term LFI memory, animals were injected with a MAPK kinase inhibitor U0126 previously used to inhibit MAPK in vivo (Lyons et al. 2006a), in ganglia and in semi-intact preparations in Aplysia (Chin et al. 2002; Sharma et al. 2003; Khabour et al. 2004). Control animals were injected with matched amounts of U0124, an inactive analog of U0126, or the vehicle DMSO. Animals were injected with either the inhibitor or vehicle 30 min prior to LFI training. No significant differences were observed in the training responses between vehicle-injected and inhibitor-treated animals, indicating that the inhibitors had no effect upon feeding behavior (Supplemental Fig. 1A). Upon testing $24 \mathrm{~h}$ later, control animals injected with vehicle alone or U0124 exhibited robust LTM as evidenced by significantly decreased response times during testing compared with training. Inhibition of MAPK activation before training completely blocked the formation of long-term LFI memory, as treated animals exhibited no significant decrease in the total response time compared with training (Fig. 1A) or in a second parameter used to assess memory, the time that the food was retained in the mouth (Supplemental Fig. 2A). To confirm these findings, similar experiments were performed with a second MEK inhibitor PD98059 that also has been used to block MAPK in vivo in Aplysia (Lyons et al. 2008) and in sensory neurons and ganglia (Bailey et al. 1997; Martin et al. 1997; Khabour et al. 2004). Animals injected with PD98059 prior to training exhibited no LTM (Fig. 1A; Supplemental Fig. 2A). These results demonstrate that MAPK signaling is necessary for long-term LFI memory.

In Aplysia, long-term sensitization and the cellular correlate long-term facilitation of presynaptic sensory neurons require a prolonged or persistent activation of MAPK (Martin et al. 1997; Sharma et al. 2003; Hu et al. 2004, 2007; Sharma and Carew 2004). In mammals, MAPK signaling is necessary for long-term molecular consolidation of memory (Schafe et al. 2000; Selcher et al. 2002; Kelly et al. 2003). To address the question of whether a more prolonged activation of MAPK is necessary for long-term LFI memory formation, animals were injected with the MEK inhibitor PD98059 immediately following training. Injection of the inhibitor after training completely blocked the formation of LTM as observed upon testing $24 \mathrm{~h}$ later (Fig. 1B; Supplemental Fig. 2B), suggesting that MAPK signaling is indeed needed for a period of time after training similar to that observed for long-term sensitization.

Although unlikely, it is possible that in the above experiments long response times during testing were observed due to long-lasting effects of the drugs in vivo. Potentially if PD98059 persisted in the animal for $>24 \mathrm{~h}$, the long-lasting inhibition of the MAPK signaling cascade could affect feeding behavior through a mechanism separate from memory formation resulting in the increased biting response times observed during testing in the above experiments. However, if the drug has no long-term 

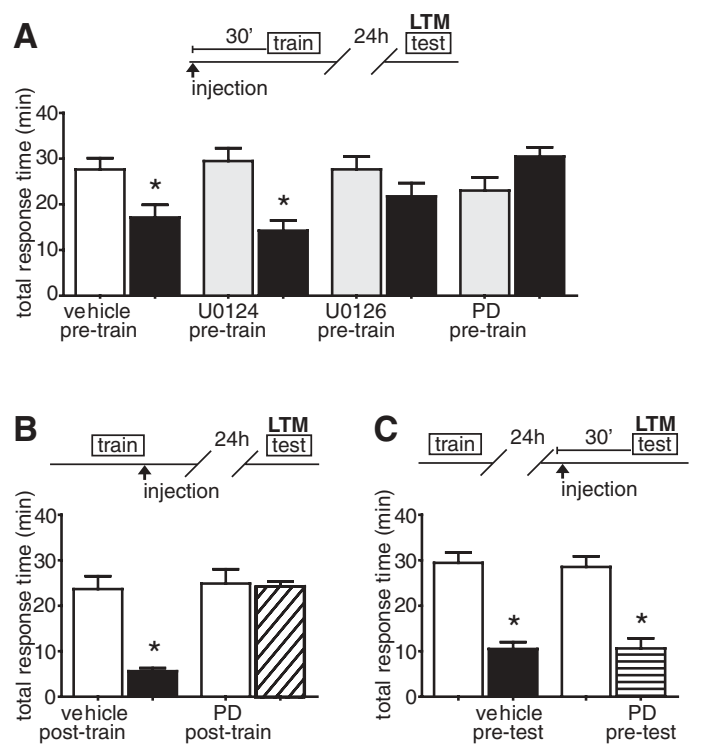

Figure 1. LTM, but not recall, is dependent upon MAPK. $(A)$ To determine if MAPK signaling was necessary for long-term LFI memory, animals were injected 30 min before training with either vehicle (DMSO, $130 \mu \mathrm{L}$ per $100 \mathrm{~g}$ body weight, $n=6)$, the MEK inhibitor U0126 $(130 \mu \mathrm{L} /$ $100 \mathrm{~g}$ body weight of $7.5 \mathrm{mM}$ stock, estimated systemic concentration $15 \mu \mathrm{M}$ in the animal, $n=6)$, the MEK inhibitor PD98059 (130 $\mu \mathrm{L}$ ) $100 \mathrm{~g}$ body weight of $7.5 \mathrm{mM}$ stock, $\sim 30 \mu \mathrm{M}$ in the animal, $n=4)$, or the inactive analog of U0126, U0124 (130 $\mu \mathrm{L} / 100 \mathrm{~g}$ body weight of $7.5 \mathrm{mM}$ stock, estimated systemic concentration $15 \mu \mathrm{M}$ in the animal, $n=8$ ). Time lines of vehicle or inhibitor injection with relationship to LFI training and testing are shown above each panel. White bars represent mean response times with SEMs for vehicle-treated animals, while gray bars signify mean response times during training for drug-treated animals. Animals were tested $24 \mathrm{~h}$ after training. Black bars represent mean response times during testing. Control animals (DMSO and U0124 groups) demonstrated significant decreases in total response time reflecting LTM, whereas both inhibitors of MAPK signaling (U0126 and PD98059) completely blocked long-term LFI memory (one-way ANOVA $\left.F_{(7,47)}=5.273 P<0.001\right)$. Asterisks represent Bonferroni multiple comparison test (MCT) post-hoc analyses with $P<0.05$ for the testing vs. training groups. $(B)$ To determine if a prolonged period of MAPK signaling was necessary for LTM, animals were injected with DMSO $(n=5)$ or PD98059 $(n=6)$ after training. Testing responses for the animals given the drug immediately after training are shown in the diagonal slashed column. Inhibition of MAPK signaling after training inhibited LTM (ANOVA $\left.F_{(3,21)}=16.10 P<0.001\right)$. (C) To test MAPK in recall, animals were trained and $24 \mathrm{~h}$ later injected with either DMSO $(n=4)$ or PD98059 ( $n=6$; testing response in horizontal lined column) $30 \mathrm{~min}$ prior to testing. Animals injected with PD98059 exhibited significant LTM similar to controls (ANOVA $F_{(3,21)}=21.09 P<0.001$ ) demonstrating that MAPK activity is not necessary for recall of LTM.

effects, then the testing session should act as a new training session in which the MEK inhibitor is not present and the animals should display STM and LTM upon further testing. To test these possibilities, animals previously injected with PD98059 and then tested $24 \mathrm{~h}$ later, for which the testing session acts as a new training session without the drug present, were tested again $30 \mathrm{~min}$ later for STM. As predicted, during the additional testing animals displayed significant memory demonstrating that PD98059 acts as a reversible inhibitor in vivo and has no effect on the animal's ability to form memory when training is performed $24 \mathrm{~h}$ after drug injection $(n=4$; training time $=$ $30.48 \mathrm{~min} \pm 2.0 ; \quad$ testing time $=5.78 \mathrm{~min} \pm 0.88$; two-tailed $t$-test $=11.28 P<0.001)$.

At the behavioral level, assessments of LTM involve the successful recall of memory. To further parse the role of MAPK signaling in LFI memory, we tested whether MAPK signaling was necessary for the recall of memory by injecting animals with PD98059 30 min prior to the 24-h memory test and comparing performance with vehicle-injected control animals. No differences were found in 24-h memory between treated and vehicle-injected control animals (Fig. 1C; Supplemental Fig. 2C), indicating that MAPK signaling is not necessary for the retrieval of LTM. Furthermore, as treated animals displayed robust memory, these experiments confirm the above experiments that PD98059 alone does not result in increased appetitive or consummatory behavioral responses.

\section{MAPK signaling is necessary for short-term LFI memory}

Mechanistically, the induction of STM and LTM vary, with STM reliant upon the activation of second messenger and kinase signaling cascades, and LTM dependent upon subsequent protein synthesis and new gene expression. Frequently, training induces the activation of early signaling pathways common to both STM and LTM. For example, following sensitization training in Aplysia serotonin induces the cAMP/PKA signaling cascade necessary in both forms of memory (Kandel 2001). However, the formation and consolidation of LTM formation often requires distinct kinase signaling pathways in addition to shared induction mechanisms with STM. This is the case seen for MAPK signaling in short- and long-term sensitization in which the activation of MAPK is necessary for long-term, but not short-term sensitization (Sharma et al. 2003), short-term synaptic plasticity in Aplysia sensory neurons (Phares and Byrne 2005), short-term conditioned taste aversion in rats (Berman et al. 1998), or short-term object recognition memory in rats (Kelly et al. 2003). In other associative learning paradigms in mammals, early activation of MAPK (ERK1/2) cascades is required for the formation of STM and LTM (Igaz et al. 2006). To further dissect the role of MAPK signaling during LFI memory formation, we investigated whether STM formation in vivo required the MAPK signaling cascade. As previously, animals were injected with either U0126 or PD98059 30 min prior to training. Control animals were injected with comparable amounts of U0124 or DMSO. Animals were tested for LFI memory $30 \mathrm{~min}$ after training. In contrast to what was previously observed for sensitization, we found that inhibition of MAPK signaling blocked the formation of short-term LFI memory (Fig. 2; Supplemental Fig. 3). Control animals demonstrated significantly
A

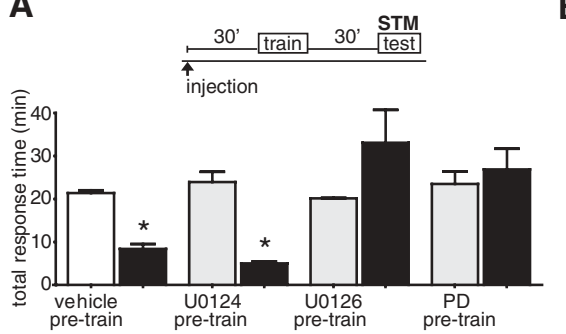

B

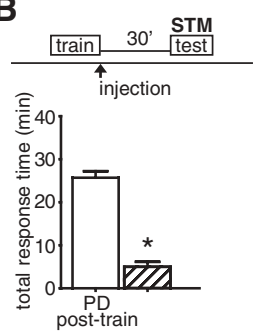

Figure 2. Short-term LFI memory is dependent upon MAPK signaling during, but not after training. (A) Animals were injected with either DMSO $(n=4)$, U0124 $(n=4)$, U0126 $(n=3)$, or PD98059 $(n=4)$ 30 min prior to LFI training at ZT 3 . Animals were tested 30 min after training. Control animals (DMSO and U0124 groups) demonstrated robust STM while animals injected with inhibitors of MAPK signaling (U0126 and PD98059) failed to exhibit STM (ANOVA $F_{(7,29)}=8.65 p<0.001$ ). Asterisks represent Bonferroni MCT post hoc-analyses with $P<0.05$ for the testing vs. training groups. (B) PD98059 injected after training had no effect on STM $(n=6$, two-tailed $t$-test $=10.83 P<0.001)$, indicating that MAPK activity is not necessary for either the maintenance or the recall of STM. 
A

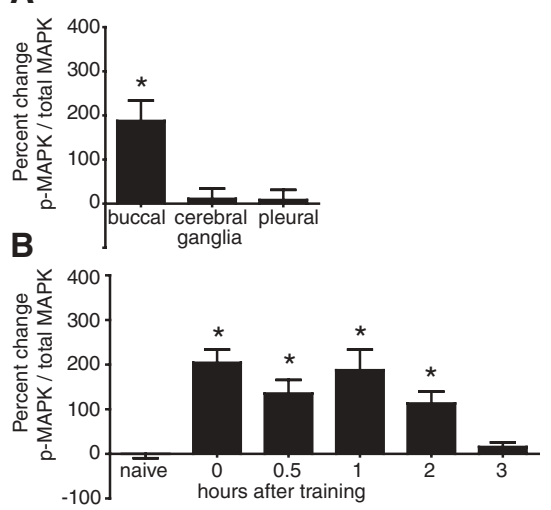

C
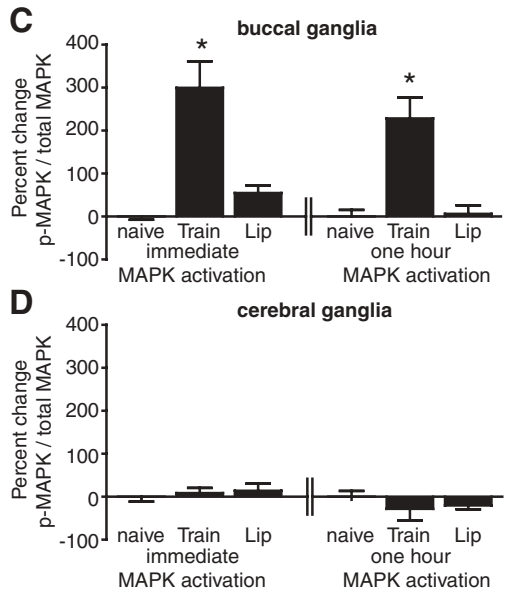

Figure 3. LFI training induces significant MAPK activation in the buccal ganglion immediately and for $2 \mathrm{~h}$ after training. Animals were trained at ZT 3, ganglia were dissected and processed, and Western blots run to measure phospho-MAPK and total MAPK levels. Quantification of P-MAPK was normalized to total MAPK. Naïve animals served as controls. Data is plotted as the mean percent change between trained animals and naïve animals. ( $A$ ) LFI training resulted in significant increases in MAPK activation in the buccal, but not the cerebral or pleural ganglia (oneway ANOVA $\left.F_{(2,22)}=6.863 P<0.01\right)$. (B) MAPK activation was significantly increased immediately after training with greater than basal level increases observed for $2 \mathrm{~h}$ after LFI training $(n=5-11$ per experiment per group; one-way ANOVA $F_{(5,45)}=8.208 P<0.001$; Bonferroni MCT post-hoc analyses: immediate, $0.5 \mathrm{~h}, 1 \mathrm{~h}$, and $2 \mathrm{~h}$ groups are all significantly different than naïve $P<0.05$ ). (C) Buccal ganglia from individual animals were used to assess MAPK activation from naïve animals $(n=$ $12)$, immediately after training $(n=12)$, and immediately after lip stimulation $(n=12)$. Similarly, buccal ganglia from individual animals were used to assess prolonged MAPK activation from naïve animals maintained in bowls for $1 \mathrm{~h}(n=4), 1 \mathrm{~h}$ after training $(n=7)$, and $1 \mathrm{~h}$ after lip stimulation $(n=8)$. Data were analyzed within groups (immediate and $1 \mathrm{~h}$ ). LFI training significantly increased immediate MAPK activation compared with naive animals and animals receiving only lip stimulation (ANOVA $\left.F_{(2,35)}=18.24 P<0.001\right)$ and the persistent phase of MAPK activation (ANOVA $\left.F_{(2,18)}=14.32 P<0.001\right)$ compared with naïve or lip stimulated animals. $(D)$ As in $C$, cerebral ganglia protein extracts were analyzed using Western blotting to determine levels of MAPK activation. No significant differences were seen in the cerebral ganglia between groups when animals were dissected immediately after training or lip stimulation (ANOVA $\left.F_{(2,35)}=0.28 P=0.75\right)$. No significant differences were observed in phospho-MAPK levels between naive and the 1-h post-training or lip stimulation groups (ANOVA $F_{(2,20)}=0.67 P=0.52$ ).

decreased total response times (Fig. 2A) and cumulative time the food was in the mouth (Supplemental Fig. 3A), while animals treated with MEK inhibitors prior to training showed no decrease in response times. These results indicate that MAPK signaling is necessary for both STM and LTM for LFI, and suggest that activation of MAPK occurs as an early step during the induction of LFI memory.

We tested whether prolonged MAPK signaling was needed for the maintenance or recall of STM by injecting PD98059 in animals immediately after training and then testing the animals 30 min later. Inhibiting MAPK signaling immediately after training did not affect STM with treated animals exhibiting significantly decreased response times upon testing (Fig. 2B; Supplemental Fig. 3B), whereas in the previous experiments it completely blocked LTM (Fig. 1B). These results suggest that MAPK signaling is involved in the early induction steps of LFI memory common to both STM and LTM formation, with a second or more prolonged phase of MAPK activation necessary for LTM.

\section{LFI training increases MAPK activation in the buccal, but not cerebral or pleural ganglia}

Based upon the above pharmacological experiments, LFI training appears to induce two phases of MAPK signaling: an early phase of MAPK signaling necessary for STM, and a more prolonged phase of MAPK signaling necessary for LTM. To further understand the role of MAPK signaling in LFI memory, we investigated the cellular location and the duration of MAPK signaling induced by LFI training. Animals received LFI training at ZT 3. Buccal, cerebral, and pleural ganglia were dissected $1 \mathrm{~h}$ post-training. In order to minimize interanimal variability with regard to baseline levels of MAPK signaling, ganglia were pooled between three animals to prepare protein for each sample. Using Western blot analysis and commercially available antibodies as previously described (Lyons et al. 2006a) we assessed MAPK activity by measuring phospho-MAPK and total MAPK levels in protein preparations from ganglia dissected from trained animals compared with ganglia dissected from naïve animals. LFI training significantly increased MAPK activation in the buccal, but not the cerebral or pleural ganglia (Fig. 3A). These results are consistent with previous research identifying the buccal ganglia as one site of molecular plasticity for long-term LFI (Levitan et al. 2008).

To define the temporal window of training induced MAPK activation in the buccal ganglia, we performed experiments measuring phospho-MAPK levels immediately, $30 \mathrm{~min}, 1 \mathrm{~h}, 2 \mathrm{~h}$, and $3 \mathrm{~h}$ after LFI training. As previously, normalized phospho-MAPK levels in trained animals were compared with naïve animals. In the buccal ganglia, MAPK activation increased immediately after training with significant increases observed as long as $2 \mathrm{~h}$ after LFI training (Fig. 3B). Thus, LFI training induces immediate and prolonged MAPK activation in the buccal ganglia.

During LFI training, mechanical and chemosensory stimulation of the lips occurs as the seaweed is presented to the animals. Previous research has demonstrated that lip stimulation is insufficient for either short- or long-term LFI memory formation (Susswein and Schwarz 1983; Schwarz et al. 1988; Katzoff et al. 2006). However, lip stimulation alone may be sufficient to induce some of the molecular changes associated with LFI memory. In Lymnaea, presentation of either the conditioned stimulus or the unconditioned stimulus alone is sufficient to induce MAPK activation in cerebral and buccal ganglia extracts as well as in lip tissue (Ribeiro et al. 2005). To determine whether the increased MAPK activation resulted solely from sensory stimulation during training or whether the observed increases comprised part of the mechanism involved in memory formation, phospho-MAPK levels were assessed in animals that received either lip stimulation alone or complete LFI training. Buccal ganglia from individual animals were dissected immediately after training to assess the initial phase of MAPK activation or $1 \mathrm{~h}$ after training to assess the later phase of MAPK activation. Naïve animals served as 
controls. As previously observed in ganglia from pooled animals, LFI training resulted in significantly increased MAPK activation in the buccal ganglia (Fig. 3C). LFI training significantly increased both the early phase of MAPK activation and the later phase of MAPK activation when compared with animals that received 20 min of lip stimulation alone (Fig. 3C). Lip stimulation by itself resulted in a small significant increase in immediate phosphoMAPK in the buccal ganglia (two-tailed Student's $t$-test, $P<$ 0.05), but did not induce the prolonged phase of MAPK activation. These results suggest that the chemosensory and mechanical cues received during lip stimulation alone are responsible for an immediate low-level induction of the MAPK signaling cascade. However, the increase in immediate MAPK activation due to lip stimulation was significantly lower than the LFI training induced increase in MAPK activation (Fig. 3C; ANOVA $P<$ 0.001; Bonferroni post-hoc comparison between the immediate increase in trained animals and that observed following lip stimulation $P<0.01$ ). Thus, while the presentation of chemosensory or mechanical stimuli during lip stimulation resulted in low level immediate activation of MAPK signaling in the buccal ganglia, the immediate high amplitude MAPK signaling, and the prolonged phase of MAPK activity required complete LFI training.

In Lymnaea, presentation of either the conditioned stimulus, the unconditioned stimulus, or both results in increased MAPK signaling within neurons in the cerebral ganglion (Ribeiro et al. 2005). We tested whether lip stimulation increased MAPK activation in the cerebral ganglion. As previously, no significant differences were observed between levels of phospho-MAPK in the cerebral ganglion from individually trained animals compared with naïve animals either immediately or $1 \mathrm{~h}$ after training (Fig. 3D). Lip stimulation did not significantly affect levels of phospho-MAPK in cerebral ganglia either. Thus, both the early phase and the protracted phase of MAPK signaling in the buccal, but not cerebral ganglia, appear to be an integral component of LFI memory formation.

\section{PKG signaling is necessary for LTM, but not STM, for LFI}

Based upon the above experiments, LFI training resulted in MAPK signaling in the buccal ganglia at multiple steps following LFI training: (1) low-level MAPK activation induced by lip stimulation alone, (2) immediate MAPK activation and signaling at a common juncture between STM and LTM, and (3) protracted MAPK signaling necessary for LTM. During LFI training, conveyance of the unconditioned stimulus, attempted swallowing associated with the lack of food reaching the gut, has been suggested to be mediated in both STM and LTM through the NO-cGMP pathway (Katzoff et al. 2006, 2010). In mammals, NO-cGMP signaling plays a critical role in associative memory and long-term potentiation (reviewed in Hawkins et al. 1998), while in invertebrates, NO signaling facilitates synaptic plasticity in many types of learning (Kemenes et al. 2002; Matsumoto et al. 2006; Antonov et al. 2007; Dacher and Gauthier 2008). One downstream effect of the NO-cGMP second-messenger cascade can be the activation of PKG (also known as cGMP-dependent protein kinase), which has been shown necessary for long-term potentiation (Zhuo et al. 1994; Lu et al. 1999), contextual fear conditioning (Ota et al. 2008, 2010a, 2010b), and passive avoidance tasks (Edwards et al. 2002). To determine if PKG signaling was necessary for shortterm LFI memory, animals were injected with KT5823, a reversible, specific cell-permeable inhibitor of PKG that has previously been used to block PKG in Aplysia studies (Lewin and Walters 1999), prior to LFI training at ZT 3. No significant difference was observed in training responses between vehicle-injected and inhibitor-treated animals (Supplemental Fig. 1B). Animals were tested 30 min after training. Both control animals and KT5823treated animals demonstrated significant decreases in total response time and cumulative time the food was in the mouth indicating robust STM (Fig. 4A; Supplemental Fig. 4A). Thus, PKG activity was not necessary for short-term LFI memory.

To determine if PKG signaling was necessary for LTM, animals were injected with either KT5823 or vehicle (ASW) $30 \mathrm{~min}$ prior to LFI training. Control animals demonstrated robust memory upon testing $24 \mathrm{~h}$ later with significant decreases observed in total response time (Fig. 4B; Supplemental Fig. 4B). In contrast, KT5823 injected prior to training completely blocked long-term LFI memory (Fig. 4B). Baseline training responses between control and experimental animals did not significantly differ, indicating that the effect of the drug was not due to nonspecific effects in the animal's response to the netted seaweed (Supplemental Fig. 1B). We also tested whether PKG activity was necessary for the recall of LTM by training animals and then injecting them with KT5823 $30 \mathrm{~min}$ prior to testing $24 \mathrm{~h}$ later. Similar to the results observed for the MAPK pathway, PKG activity was not necessary for the recall of LTM as both treated animals and vehicle-injected control animals displayed significant LTM upon testing (Fig. 4C; Supplemental Fig. 4C). These results suggest that the PKG activity necessary for LFI memory lies on a pathway exclusive to LTM formation.

\section{PKG signaling is required for the prolonged phase of MAPK signaling}

In the above experiments, PKG was identified as necessary for LTM, but not STM. Recent research has linked PKG signaling to MAPK signaling in the amygdala during fear conditioning in mice (Ota et al. 2008, 2010a, 2010b). In Aplysia, PKG phosphorylation is necessary for MAPK activation in long-term hyperexcitability (Sung et al. 2004). Thus, we hypothesized that PKG activity may be necessary for the prolonged phase of MAPK signaling induced by LFI training, but not the immediate activation of MAPK. To test this hypothesis, animals were injected with KT5823 30 min prior to LFI training. Buccal ganglia from individual animals were dissected either immediately after training to assess the early phase of MAPK activation or $1 \mathrm{~h}$ after training to assess the later phase of MAPK activation. Naïve animals served as controls. The early phase of MAPK activation induced by LFI training was not blocked by inhibition of PKG activity (Fig. 5A). Animals treated with KT5823 showed significantly increased levels of phospho-MAPK in the buccal ganglia immediately after LFI training similar to the levels in trained animals (Fig. 5A). In both groups of trained animals, LFI training induced significant increases in phospho-MAPK levels in the buccal ganglia compared with naïve animals. In animals that received similar length exposures to KT5823 but no training, the inhibitor alone appeared to slightly decrease, although not statistically significant, phosphoMAPK levels compared with naive animals. Thus, PKG activity is not necessary for the early increase in MAPK activation observed in the buccal ganglia immediately after training.

In contrast, inhibition of PKG activity significantly decreased the levels of phospho-MAPK during the extended phase of MAPK signaling in trained animals (Fig. 5B). In animals injected with KT5823 prior to training, the level of phospho-MAPK in buccal ganglia dissected $1 \mathrm{~h}$ after training was approximately $40 \%$ of the levels of phospho-MAPK in trained animals that did not receive the drug. The significant decrease in the later phase of MAPK activity was not due to an effect of KT5823 on basal MAPK activity levels as the levels of phospho-MAPK were not significantly different in animals that received KT5823 alone relative to naïve animals. Thus, PKG appears to act upstream of MAPK and 


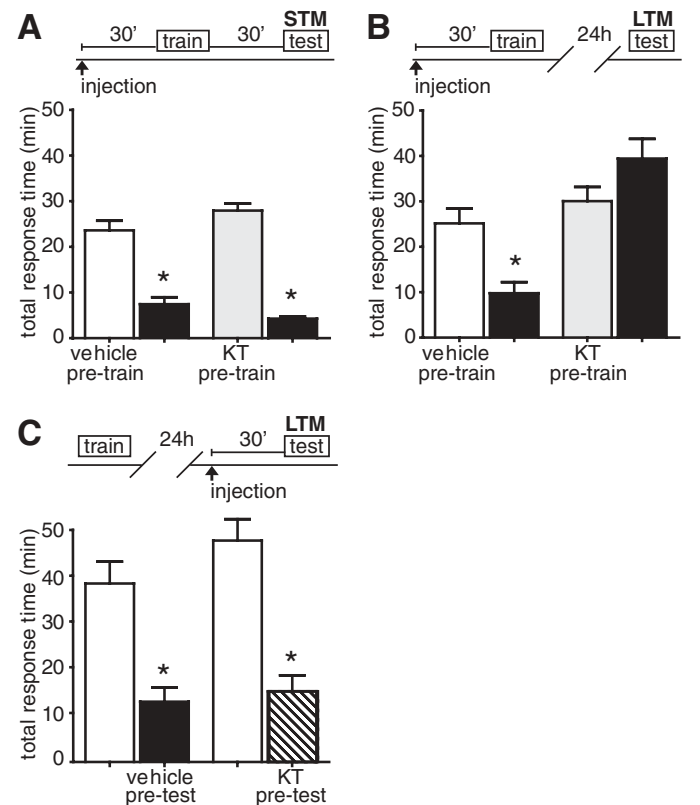

Figure 4. PKG is necessary for long-term LFI memory but not STM. (A) To determine if PKG signaling was necessary for STM, animals were injected with the PKG inhibitor KT5823 $(1 \mathrm{~mL}, 6.5 \mu \mathrm{M} / 100 \mathrm{~g}$ weight, estimated systemic concentration $100 \mathrm{nM}$ in the animal, $n=6$ ) or vehicle (ASW, $1 \mathrm{~mL} / 100 \mathrm{~g}$ weight, $n=5$ ) 30 min prior to LFI training at ZT 3. Baseline training responses between control and experimental animals were not significantly different. Vehicle-injected animals and KT5823-treated animals demonstrated significant STM (ANOVA $F_{(3,21)}=$ $65.87 P<0.001)$. Asterisks represent significant differences in Bonferroni MCT post-hoc analyses between training and testing, $P<0.05$. (B) To determine if protein kinase $G$ signaling was necessary for long-term LFI memory, animals were injected with vehicle $(n=10)$ or KT5823 $(1 \mathrm{~mL}$, $6.5 \mu \mathrm{M} / 100 \mathrm{~g}$ weight, estimated systemic concentration $100 \mathrm{nM}$ in the animal, $n=11) 30 \mathrm{~min}$ prior to LFI training at ZT 3 . Animals were tested $24 \mathrm{~h}$ after training. Control animals demonstrated significant decreases in total response time while KT5823 completely blocked longterm LFI memory (ANOVA $F_{(3,41)}=13.10 P<0.001$ ). (C) To test whether PKG was required for recall of LTM, animals were trained and $24 \mathrm{~h}$ later injected with either ASW $(n=5)$ or KT5823 $(n=5) 30$ min prior to testing. Responses of inhibitor injected animals are designated in the slashed column. Both groups demonstrated significant LTM indicating that PKG activity is not required for the recall or retrieval of memory (ANOVA $\left.F_{(3,19)}=18.2 P<0.001\right)$.

is necessary, at least in part, for the activation of the persistent phase of MAPK after training.

\section{Activation of MAPK is not dependent upon NO}

During LFI memory formation, NO appears to signal failed swallowing attempts and represents an important component of the negative reinforcement pathway during training in both STM and LTM (Katzoff et al. 2002, 2010). The dependence of the latter phase of MAPK activation on PKG signaling, at least in part, raises the question of whether NO signaling plays multiple roles in LFI memory formation. It is possible that the activation of PKG through the induction of the NO-cGMP pathway may provide a mechanism for the increased MAPK activation necessary for LTM as has been shown for MAPK activation in the amygdala following cued fear conditioning (Ota et al. 2008, 2010b). Alternatively, PKG activation may occur through a NO-independent mechanism such as the activation of membrane-bound guanylyl cyclases (reviewed in Lucas et al. 2000), atypical invertebrate guanylyl cyclases (reviewed in Morton 2004), phosphorylation by
PKC (Hou et al. 2003), or crosstalk activation by high levels of cAMP (Burnette and White 2006; Shabb 2010). We, therefore, investigated whether the NO-cGMP-PKG pathway was necessary for training-induced MAPK activation. Initially, we verified the use of L-NAME, an inhibitor of nitric oxide synthetase (NOS), in our experiments by repeating behavioral experiments initially performed by Susswein and colleagues demonstrating that LNAME completely blocks short and long-term LFI memory (Katzoff et al. 2002). Animals were injected with L-NAME $10 \mathrm{~min}$ prior to training and then tested either $30 \mathrm{~min}$ after training for STM or $24 \mathrm{~h}$ later for LTM. No significant difference was observed in training responses between vehicle-injected and inhibitor-treated animals (Supplemental Fig. 1C). We found that inhibition of NOS with L-NAME prior to LFI training completely blocked both STM and LTM as previously reported (Fig. 6A,B; Supplemental Fig. 5), whereas control animals exhibited robust STM and LTM with significantly decreased response times observed during testing.

To determine if MAPK activation was dependent upon NO signaling, animals were injected with L-NAME $10 \mathrm{~min}$ prior to training. Either immediately after training or 1-h post- training, animals were dissected and buccal ganglia processed for analysis of phospho-MAPK levels by Western blotting. Analysis of phospho-MAPK levels in the buccal ganglia immediately after training revealed that animals injected with L-NAME exhibited significantly increased phospho-MAPK levels at levels similar to those observed in vehicle-injected animals (Fig. 6C). There was no significant difference in phospho-MAPK levels $1 \mathrm{~h}$ after LFI training between animals injected with L-NAME and vehicleinjected animals (Fig. 6D). In both groups, LFI training resulted in significant increases in MAPK activation when compared with naïve animals. In the buccal ganglia of animals injected with L-NAME that received no training, phospho-MAPK levels were not significantly different than observed in naïve animals (Fig. 6C). Thus, in these experiments neither the immediate induction of MAPK following LFI training nor the prolonged phase of MAPK activation, dependent at least in part on PKG, was dependent upon NO signaling.
A

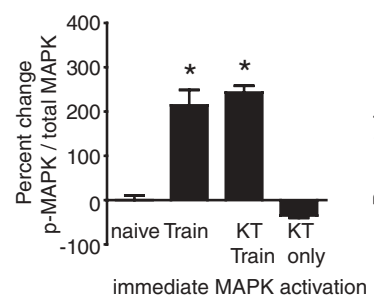

B

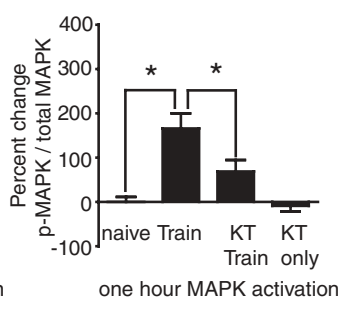

Figure 5. Inhibition of PKG significantly decreases training-induced persistent, but not immediate MAPK activation. (A) The early phase of MAPK activation induced by LFI training in buccal ganglia was not blocked by inhibition of PKG activity. Animals treated with KT5823 prior to training $(n=6)$ showed significantly increased levels of phosphoMAPK in the buccal ganglia immediately after LFI training compared with naïve animals $(n=5)$ or KT5823 injection alone $(n=7)$ similar to the levels in trained control animals $\left(n=5\right.$; ANOVA $F_{(3,22)}=65.87 P<$ 0.001 ). Asterisks represent $P<0.05$ Bonferroni post-hoc analyses for trained KT5823 and trained animals compared with naïve or drug-alone groups. (B) The persistent phase of MAPK activation induced by LFI training was significantly decreased by inhibition of PKG activity. Animals receiving LFI training $(n=11)$ exhibited significantly higher levels of activated MAPK in buccal ganglia $1 \mathrm{~h}$ after training compared with animals treated with KT5823 prior to training $(n=12)$, naïve animals $(n=10)$, or KT5823 injections $(n=10)$ without training (ANOVA $F_{(3,42)}=11.18$ $P<0.001$, Bonferroni post-hoc analyses). 
A
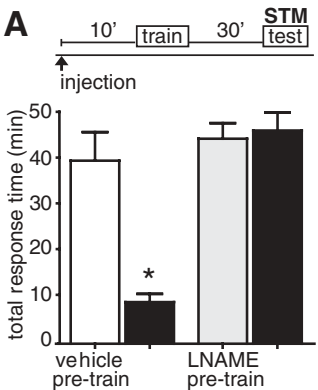

C

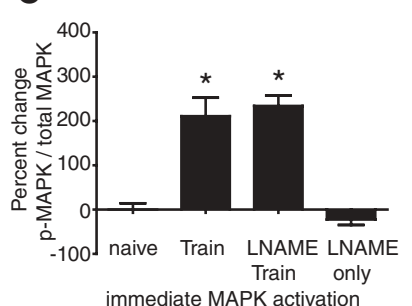

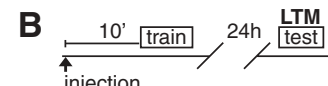

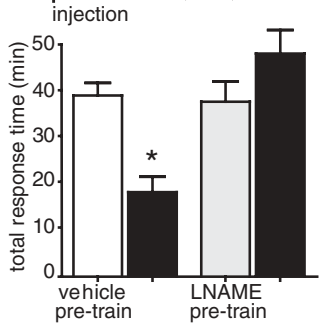

D

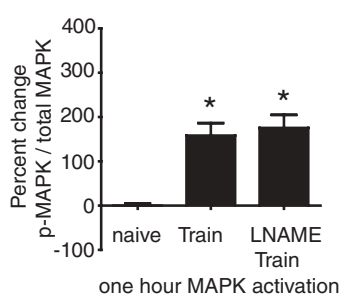

Figure 6. NOS inhibition blocks LFI memory but does not affect training-induced MAPK activation. $(A)$ Injection of the NOS inhibitor L-NAME $(10 \mathrm{mg} / \mathrm{mL}$ per $100 \mathrm{~g}$ body weight, estimated systemic drug concentration $570 \mathrm{nM} ; n=11) 10$ min prior to training blocked STM while control animals exhibited significant STM $\left(n=6\right.$; ANOVA $F_{(3,33)}=$ 15.05, $P<0.001)$. (B) Injection of L-NAME $(n=14)$ prior to training blocked LTM while control animals $(n=9)$ exhibited robust memory (ANOVA $\left.F_{(3,45)}=7.314, P<0.001\right)$. (C) Immediate MAPK activation in buccal ganglia induced by LFI training was not blocked by L-NAME. Animals treated with L-NAME prior to training $(n=4)$ showed significantly increased levels of phospho-MAPK in the buccal ganglia immediately after LFI training compared with naïve animals $(n=4)$ or L-NAME injection alone $(n=5)$ comparable to the levels in trained control $(n=$ 5) animals (ANOVA $F_{(3,19)}=26.27, P<0.001$ ). Asterisks represent $P<$ 0.05 Bonferroni post-hoc analyses for L-NAME-trained animals and trained animals compared with naïve or drug-alone groups. (D) L-NAME had no effect on MAPK 1 - $\mathrm{h}$ after training. Animals treated with L-NAME prior to training $(n=5)$ or animals receiving only training $(n=5)$ showed significantly increased levels of phospho-MAPK in the buccal ganglia $1 \mathrm{~h}$ after LFI training compared with naïve animals ( $n=$ 4; ANOVA $\left.F_{(2,13)}=13.37, P<0.01\right)$.

\section{Discussion}

In the current study, we investigated the role of MAPK and PKG signaling in memory using a negatively reinforced operant learning paradigm. Aplysia feeding behavior represents an excellent system for studying neural plasticity. Anatomically, the neural circuitry mediating plasticity in consummatory feeding behaviors lies within the buccal and cerebral ganglia, while motor neurons within the cerebral, pleural, and pedal ganglia innervate muscles controlling appetitive movements including head-lifting and head-waving (reviewed in Elliott and Susswein 2002). Through in vivo pharmacological studies, we found that MAPK signaling was necessary for both short- and long-term LFI memory. To date, the MAPK signaling cascade is the first kinase pathway shown necessary for short-term LFI memory, as previous work concluded that PKA and PKC were necessary for long-term, but not short-term LFI memory (Michel et al. 2011). The requirement for MAPK in short-term LFI stands in contrast to that observed for nonassociative memory in Aplysia in which MAPK is required for intermediate and LTM, but not short-term sensitization (Martin et al. 1997; Michael et al. 1998; Sharma et al. 2003). However, recently MAPK has been shown to acutely mediate BDNF or TGF- $\beta 1$ enhanced neurotransmitter release (Jovanovic et al. 2000; Chin et al. 2002). Combined with our results, this suggests

that the role of MAPK in STM might be broader than previously realized.

LFI training increased MAPK activation immediately after training in the buccal ganglia and for an extended period lasting $2 \mathrm{~h}$. Training induced changes in gene expression for the immediate early gene $A p C / E B P$ also localize to buccal ganglia (Levitan et al. 2008), suggesting that buccal neurons represent an important site of plasticity in LTM. Our experiments significantly extend these results by demonstrating that buccal neurons appear to be the primary site of plasticity for STM and LTM for negatively reinforced operant learning. In classical conditioning, pairing of stimuli induces changes in excitability in buccal neurons B31/32 and B51 (Lechner et al. 2000; Baxter and Byrne 2006; Lorenzetti et al. 2006). B51 also represents a single-cell correlate for operant reward conditioning (Lorenzetti et al. 2008; Mozzachiodi et al. 2008). Thus, buccal ganglia neurons exhibit a high degree of plasticity in classical conditioning, positively reinforced operant conditioning, and as seen here, negatively reinforced operant conditioning.

The olfactory and taste cues received by chemosensory neurons during lip stimulation replicate the sensory signals the animal receives during LFI training. Lip stimulation alone induced a relatively small, albeit significant, increase in immediate MAPK activation. Somewhat surprisingly, these changes occurred in buccal rather than cerebral ganglia as the cerebral ganglion contains neurons that innervate the lips, neurons receptive to chemosensory and mechanosensory inputs, and command neurons responsible for feeding behaviors associated with food arousal and consummatory movements (Xin and Kupfermann 1995; Elliott and Susswein 2002; Baxter and Byrne 2006). The induction of MAPK signaling by sensory stimulation has also been observed in Lymnaea (Ribeiro et al. 2005). Potentially, the small increase in MAPK activation we observed after lip stimulation constitutes a component of short-term plasticity. As we have observed and as previously reported (Schwarz et al. 1988), sustained lip stimulation results in a transient nonresponsiveness to the specific seaweed used for lip stimulation. Nonetheless, complete LFI training significantly increased MAPK activation by severalfold compared with lip stimulation, suggesting that MAPK signaling comprises a core component of LFI memory formation.

LFI training induced a prolonged necessary phase of MAPK signaling in the buccal ganglia dependent, at least in part, on PKG activity. Pharmacological inhibition of PKG blocked the formation of LTM and significantly decreased the later phase of MAPK activity. In contrast, inhibition of PKG had no effect on STM or the immediate activation of MAPK. PKG can directly phosphorylate MAPK and promote MAPK nuclear entry in Aplysia as shown for long-term hyperexcitability in sensory neurons after injury (Sung et al. 2004). Correspondingly in mammals, PKG promotes nuclear entry of ERK and is necessary for ERK driven gene expression after fear conditioning (Ota et al. 2010b). Presumably, nuclear entry of MAPK is required for long-term LFI to enable CREB-mediated gene transcription and $A p C / E B P$ expression as shown for long-term facilitation and sensitization (Martin et al. 1997; Michael et al. 1998; Lyons et al. 2006a). This hypothesis is consistent with the requirement for persistent PKA activity in LFI memory formation (Michel et al. 2011). Previous studies furthermore demonstrated the induction of $A p C / E B P$ gene expression following LFI training (Levitan et al. 2008).

While PKG-linked activation of MAPK was necessary for LTM, a significant proportion of prolonged phospho-MAPK $(\sim 40 \%)$ remained after PKG inhibition. This suggests that concurrent mechanisms exist through which the later phase of MAPK activation occurs after LFI training. This premise was supported by our pharmacological experiments in which injection of the MEK inhibitor PD98059 after training blocked LTM. Thus, 
MAPK activation after training also occurs via MEK phosphorylation and is necessary for LTM in addition to PKG linked MAPK activation. Multiple upstream pathways can funnel into MEK activation of MAPK including canonical neurotrophic factor activation of receptor tyrosine kinases, independent activation of G-proteins Ras or Rap, or modulation of Ras-Raf interaction by PKA or PKC (Davis and Laroche 2006; Reissner et al. 2006; Peng et al. 2010). Thus, in LFI, MAPK may signify a critical point of convergence in long-term LFI memory similar to what has been postulated for long-term sensitization (reviewed in Sharma and Carew 2004).

While our results denote a dependence upon PKG for extended MAPK activation, these experiments do not establish direct activation of MAPK by PKG. PKG may result in MAPK activation through an intermediate effector. Downstream actions suggested for PKG include regulation of phosphodiesterases, increased probability of opening $\mathrm{BK}_{\mathrm{Ca}}$ channels, regulation of phospholipase $\mathrm{C}$, stimulation of $\mathrm{IP}_{3}$ receptor opening, upregulation of serotonin transporters, and regulation of proteins involved in vesicle trafficking (reviewed in Hofmann et al. 2009; Pearce et al. 2010). Consequently, PKG has the potential to affect neural plasticity through multiple mechanisms.

What lies upstream of PKG and MAPK activation during LFI memory? In vertebrates, activation of PKG commonly occurs through retrograde NO signaling and activation of soluble guanylyl cyclases (reviewed in Kleppisch and Feil 2009; Reaume and Sokolowski 2009). In amygdala-dependent tonal fear conditioning and LTP, the CGMP-PKG pathway acts, at least in part, via activation of MAPK (Ota et al. 2008) and was dependent on NO signaling (Ota et al. 2010a, b). NO-cGMP signaling also is important for associative memory in invertebrates (Müller 1996; Teyke 1996; Katzoff et al. 2002; Kemenes et al. 2002), but NO-cGMP may impact memory formation through PKG-independent mechanisms as seen in honeybees and crickets (Müller 2000; Matsumoto et al. 2006, 2009). We found that inhibiting NOS with L-NAME completely blocked behavioral LFI memory; however, it did not block either the immediate or the extended stages of MAPK activation. Potentially if multiple signaling pathways activated MAPK after training, this could mask detection of decreased phospho-MAPK levels with NOS inhibition. However, we find this possibility unlikely since PKG inhibition significantly reduced late phase activation of MAPK as observed using the same techniques. These results suggest that the primary function of NO in LFI memory formation does not occur through PKG. Additional support for this hypothesis arises from the finding that STM was dependent upon NO but not PKG. Thus, in invertebrates, while there appears to be an evolutionarily conserved role for PKGdependent MAPK activation in learning, there appear to be fundamental differences between vertebrates and invertebrates in the mechanisms through which PKG activation may occur.

$\mathrm{NO}$ acts as a key neurotransmitter in signaling the failure to swallow during LFI training (Katzoff et al. 2002, 2010), suggesting that NO functions as a component of the unconditioned stimulus pathway. The role of NO in the unconditioned stimulus (US) pathway is bolstered by studies using lip stimulation in which exogenously applied NO can convert the normally ineffective training paradigm to an effective paradigm (Katzoff et al. 2002). NOcGMP signaling in the US pathway could occur through multiple scenarios such as cGMP activation of the cAMP pathway and PKA (Müller 2000; Matsumoto et al. 2006, 2009), the opening of ion channels or the regulation of phosphodiesterases (reviewed in Edwards et al. 2002; Kleppisch and Feil 2009). As NO is required for both short- and long-term LFI memory, we hypothesize that NO-cGMP may activate cyclic nucleotide gated ion channels to immediately affect synaptic plasticity followed by perhaps an additional role for cGMP in the activation of other signaling pathways in long-term memory. Alternatively, in LTM, a cGMP coupled adenylyl cyclase could act as a coincidence detector for the US and conditioned stimulus (CS) paths. Adenylyl cyclase appears to occupy a central point of convergence for many forms of learning including olfactory conditioning in Drosophila (reviewed in Davis 2005) and operant reward conditioning in Aplysia (Baxter and Byrne 2006; Lorenzetti et al. 2008).

If the possibility of NO-cGMP activation of the PKG linked MAPK pathway is unlikely, other mechanisms must account for activation of PKG following LFI training. Potentially, PKG could be activated by high levels of cAMP through crossactivation (Burnette and White 2006; Shabb 2010), or direct activation of PKG could occur through PKC as has been shown in cell culture studies (Hou et al. 2003). Undoubtedly, this question will be of considerable interest in future studies.

In long-term LFI memory, the prolonged phase of MAPK activation represents an integral intersection for multiple signaling pathways. In long-term LFI memory, MAPK activation appears dependent on both PKG activation and more canonical MEK activation pathways. Thus, in LFI memory, MAPK may represent a molecular node capable of integrating convergent inputs and potentially modulating divergent downstream effectors as postulated for sensitization (Reissner et al. 2006). Intriguingly, while great similarity exists between the role of MAPK in LFI memory and long-term sensitization, the requirement for MAPK for STM in LFI is strikingly different than observed for sensitization. As recent research discounted the involvement of either PKA or PKC in short-term LFI memory, MAPK signaling represents one of, if not the primary, kinase cascade through which short-term LFI memory formation occurs. The results presented here have highlighted the multiple roles of MAPK in memory formation. Overall, we have shown that MAPK signaling is a key component in short- and long-term LFI memory and that even highly conserved signaling pathways may be differentially recruited and fulfill diverse roles between learning paradigms or types of memory.

\section{Acknowledgments}

This work was supported by NIH Grants from the NINDS, NS050589 to A.E. and by NIMH Grant MH081012 to L.C.L. The authors thank Jacob Gardner for his assistance in animal maintenance and Dr. Kim van der Linde for thoughtful discussions.

\section{References}

Antonov I, Ha T, Antonova I, Moroz LL, Hawkins RD. 2007. Role of nitric oxide in classical conditioning of siphon withdrawal in Aplysia. $J$ Neurosci 27: 10993-11002.

Atkins CM, Selcher JC, Petraitis JJ, Trzaskos JM, Sweatt JD. 1998. The MAPK cascade is required for mammalian associative learning. Nat Neurosci 1: 602-609.

Bailey CH, Kaang BK, Chen M, Martin KC, Lim CS, Casadio A, Kandel ER. 1997. Mutation in the phosphorylation sites of MAP kinase blocks learning-related internalization of apCAM in Aplysia sensory neurons. Neuron 18: 913-924.

Bailey CH, Barco A, Hawkins RD, Kandel ER. 2008. Molecular studies of learning and memory in Aplysia and the hippocampus: A comparative analysis of implicit and explicit memory storage. In Learning and memory: A comprehensive reference (ed. JH Byrne), pp. 11-29. Academic Press, Oxford, UK.

Baxter DA, Byrne JH. 2006. Feeding behavior of Aplysia: A model system for comparing cellular mechanisms of classical and operant conditioning. Learn Mem 13: 669-680.

Berman DE, Hazvi S, Rosenblum K, Seger R, Dudai Y. 1998. Specific and differential activation of mitogen-activated protein kinase cascades by unfamiliar taste in the insular cortex of the behaving rat. J Neurosci 18: 10037-10044.

Botzer D, Markovich S, Susswein AJ. 1998. Multiple memory processes following training that a food is inedible in Aplysia. Learn Mem 5: $204-219$. 
Burnette JO, White RE. 2006. PGI2 opens potassium channels in retinal pericytes by cyclic AMP-stimulated, cross-activation of PKG. Exp Eye Res 83: $1359-1365$.

Chin J, Angers A, Cleary LJ, Eskin A, Byrne JH. 2002. Transforming growth factor beta1 alters synapsin distribution and modulates synaptic depression in Aplysia. J Neurosci 22: RC220.

Cropper EC, Evans CG, Hurwitz I, Jing J, Proekt A, Romero A, Rosen SC. 2004. Feeding neural networks in the mollusc Aplysia. Neurosignals 13: $70-86$.

Dacher M, Gauthier M. 2008. Involvement of NO-synthase and nicotinic receptors in learning in the honey bee. Physiol Behav 95: 200-207.

Davis RL. 2005. Olfactory memory formation in Drosophila: From molecular to systems neuroscience. Annu Rev Neurosci 28: 275-302.

Davis S, Laroche S. 2006. Mitogen-activated protein kinase/extracellular regulated kinase signalling and memory stabilization: A review. Genes Brain Behav 5 (Suppl. 2): 61-72.

Edwards TM, Rickard NS, Ng KT. 2002. Inhibition of guanylate cyclase and protein kinase $\mathrm{G}$ impairs retention for the passive avoidance task in the day-old chick. Neurobiol Learn Mem 77: 313-326.

Elliott CJ, Susswein AJ. 2002. Comparative neuroethology of feeding control in molluscs. J Exp Biol 205: 877-896.

English JD, Sweatt JD. 1997. A requirement for the mitogen-activated protein kinase cascade in hippocampal long term potentiation. J Biol Chem 272: 19103-19106.

Hawkins RD, Son H, Arancio O. 1998. Nitric oxide as a retrograde messenger during long-term potentiation in hippocampus. Prog Brain Res 118: 155-172.

Hofmann F, Bernhard D, Lukowski R, Weinmeister P. 2009. cGMP regulated protein kinases (cGK). Handb Exp Pharmacol 191: 137-162.

Hou Y, Lascola J, Dulin NO, Ye RD, Browning DD. 2003. Activation of cGMP-dependent protein kinase by protein kinase C. J Biol Chem 278: 16706-16712.

Hu JY, Glickman L, Wu F, Schacher S. 2004. Serotonin regulates the secretion and autocrine action of a neuropeptide to activate MAPK required for long-term facilitation in Aplysia. Neuron 43: 373-385.

Hu JY, Chen Y, Schacher S. 2007. Protein kinase C regulates local synthesis and secretion of a neuropeptide required for activity-dependent long-term synaptic plasticity. J Neurosci 27: 8927-8939.

Igaz P, Salvi R, Rey JP, Glauser M, Pralong FP, Gaillard RC. 2006. Effects of cytokines on gonadotropin-releasing hormone $(\mathrm{GnRH})$ gene expression in primary hypothalamic neurons and in GnRH neurons immortalized conditionally. Endocrinology 147: 1037-1043.

Jovanovic JN, Czernik AJ, Fienberg AA, Greengard P, Sihra TS. 2000. Synapsins as mediators of BDNF-enhanced neurotransmitter release. Nat Neurosci 3: 323-329.

Kandel ER. 2001. The molecular biology of memory storage: A dialogue between genes and synapses. Science 294: 1030-1038.

Katzoff A, Ben-Gedalya T, Susswein AJ. 2002. Nitric oxide is necessary for multiple memory processes after learning that a food is inedible in Aplysia. J Neurosci 22: 9581-9594.

Katzoff A, Ben-Gedalya T, Hurwitz I, Miller N, Susswein YZ, Susswein AJ. 2006. Nitric oxide signals that Aplysia have attempted to eat, a necessary component of memory formation after learning that food is inedible. J Neurophysiol 96: 1247-1257.

Katzoff A, Miller N, Susswein AJ. 2010. Nitric oxide and histamine signal attempts to swallow: A component of learning that food is inedible in Aplysia. Learn Mem 17: 50-62.

Kelly A, Laroche S, Davis S. 2003. Activation of mitogen-activated protein kinase/extracellular signal-regulated kinase in hippocampal circuitry is required for consolidation and reconsolidation of recognition memory. J Neurosci 23: 5354-5360.

Kemenes I, Kemenes G, Andrew RJ, Benjamin PR, O'Shea M. 2002. Critical time-window for NO-cGMP-dependent long-term memory formation after one-trial appetitive conditioning. I Neurosci 22: 1414-1425.

Khabour O, Levenson J, Lyons LC, Kategaya LS, Chin J, Byrne JH, Eskin A. 2004. Coregulation of glutamate uptake and long-term sensitization in Aplysia. J Neurosci 24: 8829-8837.

Kleppisch T, Feil R. 2009. cGMP signalling in the mammalian brain: Role in synaptic plasticity and behaviour. Handb Exp Pharmacol 191: 549-579.

Lechner HA, Baxter DA, Byrne JH. 2000. Classical conditioning of feeding in Aplysia: II. Neurophysiological correlates. J Neurosci 20: 3377-3386.

Levitan D, Lyons LC, Perelman A, Green CL, Motro B, Eskin A, Susswein AJ. 2008. Training with inedible food in Aplysia causes expression of $\mathrm{C} / \mathrm{EBP}$ in the buccal but not cerebral ganglion. Learn Mem 15: $412-416$.

Lewin MR, Walters ET. 1999. Cyclic GMP pathway is critical for inducing long-term sensitization of nociceptive sensory neurons. Nat Neurosci 2: $18-23$.
Lorenzetti FD, Mozzachiodi R, Baxter DA, Byrne JH. 2006. Classical and operant conditioning differentially modify the intrinsic properties of an identified neuron. Nat Neurosci 9: 17-19.

Lorenzetti FD, Baxter DA, Byrne JH. 2008. Molecular mechanisms underlying a cellular analog of operant reward learning. Neuron 59: $815-828$.

Lu YF, Kandel ER, Hawkins RD. 1999. Nitric oxide signaling contributes to late-phase LTP and CREB phosphorylation in the hippocampus. J Neurosci 19: 10250-10261.

Lucas KA, Pitari GM, Kazerounian S, Ruiz-Stewart I, Park J, Schulz S, Chepenik KP, Waldman SA. 2000. Guanylyl cyclases and signaling by cyclic GMP. Pharmacol Rev 52: 375-414.

Lyons LC, Rawashdeh O, Katzoff A, Susswein AJ, Eskin A. 2005. Circadian modulation of complex learning in diurnal and nocturnal Aplysia. Proc Natl Acad Sci 102: 12589-12594.

Lyons LC, Collado MS, Khabour O, Green CL, Eskin A. 2006a. The circadian clock modulates core steps in long-term memory formation in Aplysia. J Neurosci 26: 8662-8671.

Lyons LC, Rawashdeh O, Eskin A. 2006b. Non-ocular circadian oscillators and photoreceptors modulate long term memory formation in Aplysia. J Biol Rhythms 21: 245-255.

Lyons LC, Green CL, Eskin A. 2008. Intermediate-term memory is modulated by the circadian clock. J Biol Rhythms 23: 538-542.

Martin KC, Michael D, Rose JC, Barad M, Casadio A, Zhu H, Kandel ER. 1997. MAP kinase translocates into the nucleus of the presynaptic cell and is required for long-term facilitation in Aplysia. Neuron 18: 899-912.

Matsumoto Y, Unoki S, Aonuma H, Mizunami M. 2006. Critical role of nitric oxide-cGMP cascade in the formation of cAMP-dependent long-term memory. Learn Mem 13: 35-44.

Matsumoto Y, Hatano A, Unoki S, Mizunami M. 2009. Stimulation of the cAMP system by the nitric oxide-cGMP system underlying the formation of long-term memory in an insect. Neurosci Lett 467: $81-85$.

Michael D, Martin KC, Seger R, Ning MM, Baston R, Kandel ER. 1998. Repeated pulses of serotonin required for long-term facilitation activate mitogen-activated protein kinase in sensory neurons of Aplysia. Proc Natl Acad Sci 95: 1864-1869.

Michel M, Green CL, Lyons LC. 2011. PKA and PKC are required for long-term but not short-term in vivo operant memory in Aplysia. Learn Mem 18: $19-23$.

Moressis A, Friedrich AR, Pavlopoulos E, Davis RL, Skoulakis EM. 2009. A dual role for the adaptor protein DRK in Drosophila olfactory learning and memory. J Neurosci 29: 2611-2625.

Morton DB. 2004. Invertebrates yield a plethora of atypical guanylyl cyclases. Mol Neurobiol 29: 97-116.

Mozzachiodi R, Lorenzetti FD, Baxter DA, Byrne JH. 2008. Changes in neuronal excitability serve as a mechanism of long-term memory for operant conditioning. Nat Neurosci 11: 1146-1148.

Müller U. 1996. Inhibition of nitric oxide synthase impairs a distinct form of long-term memory in the honeybee, Apis mellifera. Neuron 16: $541-549$.

Müller U. 2000. Prolonged activation of cAMP-dependent protein kinase during conditioning induces long-term memory in honeybees. Neuron 27: $159-168$.

Ota KT, Pierre VJ, Ploski JE, Queen K, Schafe GE. 2008. The NO-cGMP-PKG signaling pathway regulates synaptic plasticity and fear memory consolidation in the lateral amygdala via activation of ERK/MAP kinase. Learn Mem 15: 792-805.

Ota KT, Monsey MS, Wu MS, Schafe GE. 2010a. Synaptic plasticity and NO-cGMP-PKG signaling regulate pre- and postsynaptic alterations at rat lateral amygdala synapses following fear conditioning. PLoS One 5: e11236. doi: 10.1371/journal.pone. 0011236.

Ota KT, Monsey MS, Wu MS, Young GJ, Schafe GE. 2010b. Synaptic plasticity and NO-cGMP-PKG signaling coordinately regulate ERK-driven gene expression in the lateral amygdala and in the auditory thalamus following Pavlovian fear conditioning. Learn Mem 17: 221-235.

Pagani MR, Oishi K, Gelb BD, Zhong Y. 2009. The phosphatase SHP2 regulates the spacing effect for long-term memory induction. Cell 139: 186-198.

Pearce LR, Komander D, Alessi DR. 2010. The nuts and bolts of AGC protein kinases. Nat Rev Mol Cell Biol 11: 9-22.

Peng S, Zhang Y, Zhang J, Wang H, Ren B. 2010. ERK in learning and memory: A review of recent research. Int J Mol Sci 11: 222-232.

Phares GA, Byrne JH. 2005. Analysis of 5-HT-induced short-term facilitation at Aplysia sensorimotor synapse during bursts: Increased synaptic gain that does not require ERK activation. J Neurophysiol 94: 871-877.

Purcell AL, Sharma SK, Bagnall MW, Sutton MA, Carew TJ. 2003. Activation of a tyrosine kinase-MAPK cascade enhances the induction of 
long-term synaptic facilitation and long-term memory in Aplysia. Neuron 37: 473-484.

Reaume CJ, Sokolowski MB. 2009. cGMP-dependent protein kinase as a modifier of behaviour. Handb Exp Pharmacol 423-443.

Reissner KJ, Shobe JL, Carew TJ. 2006. Molecular nodes in memory processing: Insights from Aplysia. Cell Mol Life Sci 63: 963-974.

Ribeiro MJ, Schofield MG, Kemenes I, O'Shea M, Kemenes G, Benjamin PR. 2005. Activation of MAPK is necessary for long-term memory consolidation following food-reward conditioning. Learn Mem 12: $538-545$.

Schafe GE, Atkins CM, Swank MW, Bauer EP, Sweatt JD, LeDoux JE. 2000. Activation of ERK/MAP kinase in the amygdala is required for memory consolidation of pavlovian fear conditioning. J Neurosci 20: $8177-8187$.

Schwarz M, Susswein AJ. 1992. Presence of conspecifics facilitates learning that food is inedible in Aplysia fasciata. Behav Neurosci 106: 250-261.

Schwarz M, Markovich S, Susswein AJ. 1988. Parametric features of inhibition of feeding in Aplysia by associative learning, satiation, and sustained lip stimulation. Behav Neurosci 102: 124-133.

Schwarz M, Blumberg S, Susswein AJ. 1998. Social isolation blocks the expression of memory after training that a food is inedible in Aplysia fasciata. Behav Neurosci 112: 942-951.

Selcher JC, Atkins CM, Trzaskos JM, Paylor R, Sweatt JD. 1999. A necessity for MAP kinase activation in mammalian spatial learning. Learn Mem 6: $478-490$.

Selcher JC, Weeber EJ, Varga AW, Sweatt JD, Swank M. 2002. Protein kinase signal transduction cascades in mammalian associative conditioning. Neuroscientist 8: 122-131.

Shabb JB. 2010. Cyclic nucleotide specificity and cross-activation of cyclic nucleotide receptors. In Handbook of cell signaling, Vol 2 (ed. RA Bradshaw, EA Dennis), pp. 1549-1554. Academic Press, San Diego, CA.
Sharma SK, Carew TJ. 2002. Inclusion of phosphatase inhibitors during Western blotting enhances signal detection with phospho-specific antibodies. Anal Biochem 307: 187-189.

Sharma SK, Carew TJ. 2004. The roles of MAPK cascades in synaptic plasticity and memory in Aplysia: Facilitatory effects and inhibitory constraints. Learn Mem 11: 373-378.

Sharma SK, Sherff CM, Shobe J, Bagnall MW, Sutton MA, Carew TJ. 2003. Differential role of mitogen-activated protein kinase in three distinct phases of memory for sensitization in Aplysia. J Neurosci 23: 3899-3907.

Sung YJ, Walters ET, Ambron RT. 2004. A neuronal isoform of protein kinase $\mathrm{G}$ couples mitogen-activated protein kinase nuclear import to axotomy-induced long-term hyperexcitability in Aplysia sensory neurons. J Neurosci 24: 7583-7595.

Susswein AJ, Schwarz M. 1983. A learned change of response to inedible food in Aplysia. Behav Neural Biol 39: 1-6.

Susswein AJ, Schwarz M, Feldman E. 1986. Learned changes of feeding behavior in Aplysia in response to edible and inedible foods. J Neurosci 6: $1513-1527$.

Sweatt JD. 2004. Mitogen-activated protein kinases in synaptic plasticity and memory. Curr Opin Neurobiol 14: 311-317.

Sweatt JD. 2010. Mechanisms of memory. Academic, Amsterdam, The Netherlands.

Teyke T. 1996. Nitric oxide, but not serotonin, is involved in acquisition of food-attraction conditioning in the snail Helix pomatia. Neurosci Lett 206: $29-32$.

Xin Y, Kupfermann I. 1995. Neuronal pathways mediating head turning behavior in Aplysia. Neurosci Lett 186: 197-199.

Zhuo M, Hu Y, Schultz C, Kandel ER, Hawkins RD. 1994. Role of guanylyl cyclase and cGMP-dependent protein kinase in long-term potentiation. Nature 368: 635-639.

Received November 2, 2010; accepted in revised form November 16, 2010. 


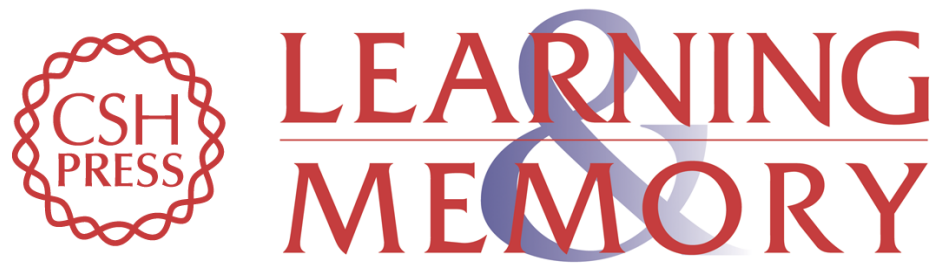

\section{PKG-mediated MAPK signaling is necessary for long-term operant memory in Aplysia}

Maximilian Michel, Charity L. Green, Arnold Eskin, et al.

Learn. Mem. 2011, 18:

Access the most recent version at doi:10.1101//m.2063611

\section{Supplemental http://learnmem.cshlp.org/content/suppl/2011/01/18/18.2.108.DC1 Material}

References This article cites 77 articles, 33 of which can be accessed free at: http://learnmem.cshlp.org/content/18/2/108.full.html\#ref-list-1

License

Email Alerting

Receive free email alerts when new articles cite this article - sign up in the box at the Service top right corner of the article or click here. 